\title{
Danggui Sini Decoction Protected Islet Endothelial Cell Survival from Hypoxic Damage via PI3K/Akt/eNOS Pathway
}

\author{
Wenting Chen (D), ${ }^{1,2}$ Caoxin Huang, ${ }^{1}$ Chen Yang, ${ }^{1}$ Xilin Ge, ${ }^{1}$ Wenfang Huang, ${ }^{1}$ Xuejun Li, \\ Shuyu Yang $\left(\mathbb{D},{ }^{1}\right.$ and Suhuan Liu $(\mathbb{1})^{1,3}$ \\ ${ }^{1}$ Xiamen Diabetes Institute, The First Affiliated Hospital of Xiamen University, Xiamen 361003, China \\ ${ }^{2}$ Medical College of Xiamen University, Xiamen 361000, China \\ ${ }^{3}$ Central Laboratory, The First Affiliated Hospital of Xiamen University, Xiamen 361003, China
}

Correspondence should be addressed to Shuyu Yang; xmyangshuyu@126.com and Suhuan Liu; liusuhuan@xmu.edu.cn

Received 24 February 2018; Revised 11 June 2018; Accepted 13 June 2018; Published 10 July 2018

Academic Editor: Yong C. Boo

Copyright (C) 2018 Wenting Chen et al. This is an open access article distributed under the Creative Commons Attribution License, which permits unrestricted use, distribution, and reproduction in any medium, provided the original work is properly cited.

\begin{abstract}
Danggui Sini decoction (DSD) is a traditional Chinese decoction, which is wildly applied and showed to be effective in ameliorating ischemia-related symptoms. However, the mechanisms of DSD action in ischemic damage remain to be fully clarified. Pancreatic islet endothelial cells are pivotal constituent of islet microvasculature, with high vulnerability to hypoxic injuries. Here, using MST1 cell, a pancreatic islet endothelial cell-line, as a model, we investigated the effects of DSD on hypoxia-stimulated endothelial cell lesions and its underlying mechanisms. We found that DSD-Containing Serum (DSD-CS), collected from DSD-treated rats, could efficiently protect MST1 survival and proliferation from Cobalt chloride $\left(\mathrm{CoCl}_{2}\right)$ induced damage, including cell viability, proliferation, and tube formation. Furthermore, DSD-CS restored the activity of PI3K/Akt/eNOS signaling inhibited by $\mathrm{CoCl}_{2}$ in MST1 cells. The protective effect of DSD-CS could be blocked by the specific PI3K/Akt/eNOS inhibitor LY294002, suggesting that DSD-CS protection of MST1 cell survival from hypoxia was mediated by PI3K/Akt/eNOS pathway. In conclusion, DSD treatment protected MST1 survival from hypoxic injuries via PI3K/Akt/eNOS pathway, indicating its role in protecting microvascular endothelial cells.
\end{abstract}

\section{Introduction}

Danggui Sini decoction (DSD), first reported in Shanghan Lun, is a commonly used traditional Chinese medicine in increasing cardiovascular and peripheral circulation [1]. In addition, DSD is also applied in treating watery diarrhea, shock, heart failure, and severe poor extremity circulation [2-5]. It is extracted from Angelica sinensis, Ramulus Cinnamomi, and Radix Puerariae for nourishing blood, antagonizing vascular diseases, and hemodynamic instability [2, $5,6]$. Angelical sinensis acts as a traditional phytochemicals, which was first reported in Shennong Bencao Jing, with ability to promote angiogenesis $[1,3,5,7]$. Ramulus Cinnamomi is a principal bioactive ingredient with antioxidant and antiinflammatory effects [8-12]. Puerarin is a traditional Chinese herbal, which shows a great effect in protecting islets cells from oxidative stress by activating antioxidant enzymes [13,
14]. In previous study, we have reported that DSD was effective in ameliorating diabetic peripheral neuropathy (DPN), one of the common and typical diabetes microvascular complications [6].

Microvascular endothelial cells form a physiologically vital interface between the circulating blood and surrounding microenvironment and thus play a key role in regulating the access of cells and blood molecules into the tissue under a variety of conditions including inflammation, repair, and survival $[15,16]$. Besides, uninterrupted blood flow through the microvasculature is critical for overall organ function to supporting nutrition and adequate oxygen [1518]. As the typical component of islet microvasculature and microcirculation [19], pancreatic islet endothelial cells are critical for oxygen and hormones transportation and, therefore, play important roles in the pancreas $\beta$-cell survival and proliferation [20-22], with high vulnerability to hypoxic 
TABLE 1: Parameters of ferulic acid, cinnamic acid, and puerarin for MS condition.

\begin{tabular}{lccccrr}
\hline Compound Name & Precursor Ion & Product Ion & Fragmentor & Collision Energy & Cell Accelerator Voltage & Polarity \\
\hline Ferulic acid & 193.2 & 134.2 & 91 & 11 & 0 & Negative \\
\hline Cinnamic acid & 147.2 & 103.2 & 83 & 5 & Negative \\
\hline Puerarin & 417.1 & 297.1 & 152 & 22 & 0 & Positive \\
\hline
\end{tabular}

injuries [23-26]. Thus the pancreatic islet endothelial cell may serve as a good model for studying microvascular endothelial cell biological activities.

Here, using a pancreatic islet endothelial cell-line MST1 cell as a microvasculature model, we designed the present study to evaluate whether Danggui Sini decoction can protect endothelial cells function and survival against hypoxic stress in vitro.

\section{Materials and Methods}

2.1. Materials. Cobalt chloride $\left(\mathrm{CoCl}_{2}\right)$, a chemical to induce hypoxia-like reactions, was purchased from Sigma-Aldrich (Merck Millipore, Darmstadt, Germany). Cell Counting Kit-8 (CCK-8) was obtained from Dojindo Laboratories (Kumamoto, Japan). In Situ Cell Death Detection Kit for TUNEL assay was obtained from Roche (Basel, Switzerland). Cell-Light ${ }^{\mathrm{TM}}$ EdU Apollo488 In Vitro Kit was obtained from Ribobio (Guangzhou, China). Matrigel Matrix Growth Factor Reduced (356230) was purchased from Corning Incorporated (Tewksbury, MA, USA). Akt, p-Akt, p-eNOS, eNOS, and $\beta$-actin antibodies were purchased from Cell Signaling Technology (Boston, MA, USA). LY294002, a PI3K inhibitor, was obtained from Cell Signaling Technology (Boston, MA, USA).

2.2. Preparation of DSD-Containing Serum (DSD-CS). The DSD was prepared by the Department of Pharmacy of the First Affiliated Hospital of Xiamen University, China. Male Sprague-Dawley rats (350-400 g) were purchased from Shanghai SLAC Laboratory Animal Co. Ltd. (Shanghai, China). All procedures conducted in the animal experiments were approved by Xiamen University Animal Care and Use Committee. Rats were divided into two groups randomly. One group was given DSD (100 mg/kg, dissolved in water) with daily gavage for 7 consecutive days, while the other group was given an equal volume of water only. On day 7 , 2 hours after the last oral administration of DSD, rats were anesthetized, and the blood was collected from abdominal aorta and kept at $4^{\circ} \mathrm{C}$ overnight. The second day, blood was centrifuged at $3000 \mathrm{rpm}$ for $10 \mathrm{~min}$ and the serum was collected. The serum was inactivated for 30 mins at $56^{\circ} \mathrm{C}$ and then kept at $-20^{\circ} \mathrm{C}$ until use.

2.3. Quality Control for DSD and DSD-Containing Serum (DSD-CS). As described in the method and material part, we have compared the chemical composition of DSD and DSDContaining Serum using mass spectrometer HPLC analysis (Figure 1). The chemical compositions of DSD itself and DSDContaining Serum (DSD-CS) were determined by an Agilent
6410 triple stage quadrupole mass spectrometer equipped with an ESI ion source and an Agilent 1290 HPLC system with autosampler (Agilent Technologies, Santa Clara, CA, USA). The analytes were separated on ACQUITY UPLC HSS C18 $(2.1 \times 100 \mathrm{~mm}, 1.8 \mu \mathrm{m})$ used at $40^{\circ} \mathrm{C}$. The mobile phase was used with a gradient elution: 0-5 $\mathrm{min}, 10-80 \% \mathrm{~B}$, and 5$7 \mathrm{~min}, 80-90 \%$, at a flow rate of $0.3 \mathrm{ml} / \mathrm{min}$. ESI-MS/MS conditions were set as follows: gas temperature $300^{\circ} \mathrm{C}$, gas flow $5 \mathrm{l} / \mathrm{min}$, capillary: positive $4000 \mathrm{~V}$, negative $3500 \mathrm{~V}$, and nebulizer pressure 45 psi. MS acquisition was performed in multiple reaction monitoring (MRM) mode. The compound dependent parameters used for analysis were summarized in Table 1. The result was shown in Figure 1. By comparing to reference standards, major peaks were identified as ferulic acid, cinnamic acid, and puerarin. DSD is the same as DSDContaining Serum (DSD-CS).

2.4. Cell Culture and Treatment. MST1 cell, an islet endothelial cell-line, was purchased from American Type Culture Collection (ATCC). MST1 were cultured in RPMI 1640 medium supplemented with $10 \%$ fetal bovine serum (FBS), streptomycin $(100 \mu \mathrm{g} / \mathrm{mL})$, and penicillin (100 Units $/ \mathrm{mL})$. Cells were exposed to $\mathrm{CoCl}_{2}$ with or without DSD-CS $(10 \%$, $\mathrm{v} / \mathrm{v}$ ) for 12 hours or 24 hours. According to the results of dose and time course tests, $\mathrm{CoCl}_{2}$ stimulation at $200 \mu \mathrm{M}$ for 24 hours was applied in the rest of the study.

2.5. Cell Viability Assay. Cell viability was evaluated by Cell Counting Kit. MST1 cells were seeded in 96-well plates (5000 cells/ well) and exposed to $\mathrm{CoCl}_{2}(200 \mu \mathrm{M})$ with or without DSD-CS for 24 hours. Then $10 \mu \mathrm{L}$ CCK- 8 reagent was added to each well of the plates and incubated at $37^{\circ} \mathrm{C}$ for 1 hour. The absorbance was measured with the Bio-Tek Synergy H1 Microplate Reader (Winooski, VT, USA) at $450 \mathrm{~nm}$.

2.6. Apoptosis Assay. Cell apoptosis was evaluated by In Situ Cell Death Detection Kit with counterstaining by DAPI. Images were taken by a fluorescence microscope (Olympus, Shanghai, China).

2.7. Cell Proliferation Assay. Cell proliferation was evaluated by Cell-Light ${ }^{\mathrm{TM}}$ EdU Apollo488 In Vitro Kit. Briefly, MST1 cells were fixed in the $4 \%$ paraformaldehyde after treatment at room temperature for $30 \mathrm{~min}$ and then stained with Apollo ${ }^{\circledR}$ for $30 \mathrm{~min}$. Nuclei were stained with Hoechst 33342 at room temperature for $30 \mathrm{~min}$. Images were taken by a fluorescence microscope (Olympus, Shanghai, China).

2.8. Tube Formation Assay. Tube formation capacity was measured by Matrigel tube formation assay. Following the 


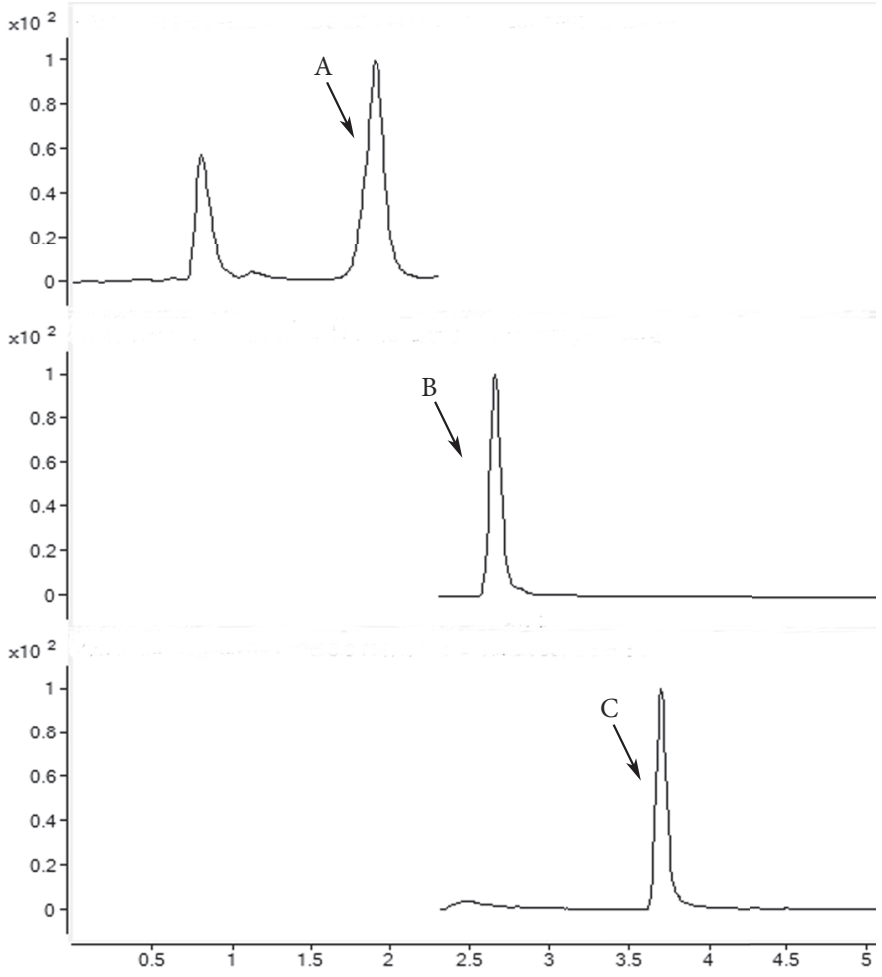

(a)
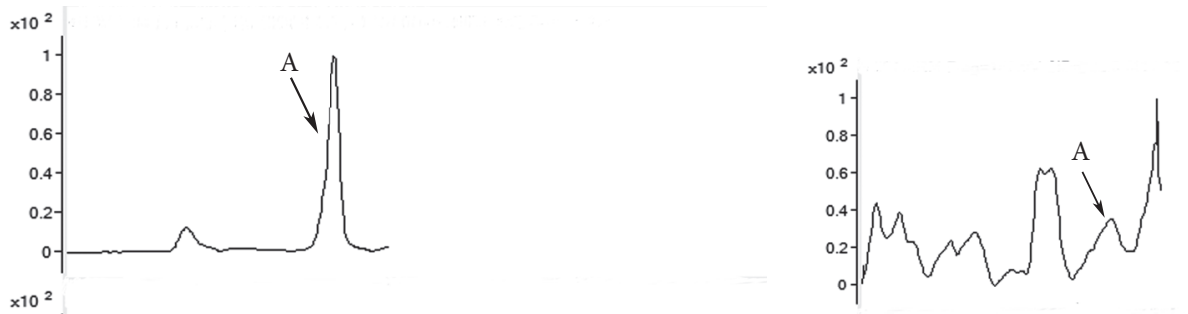

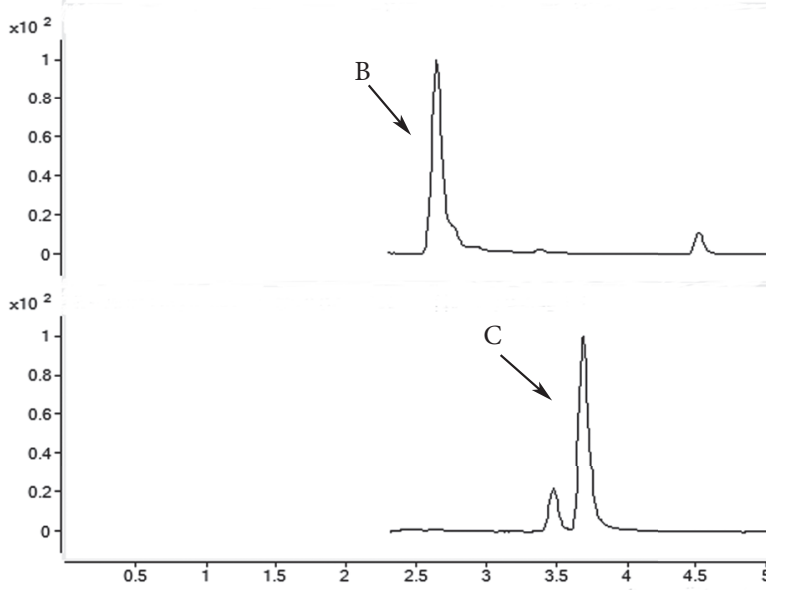

(b)
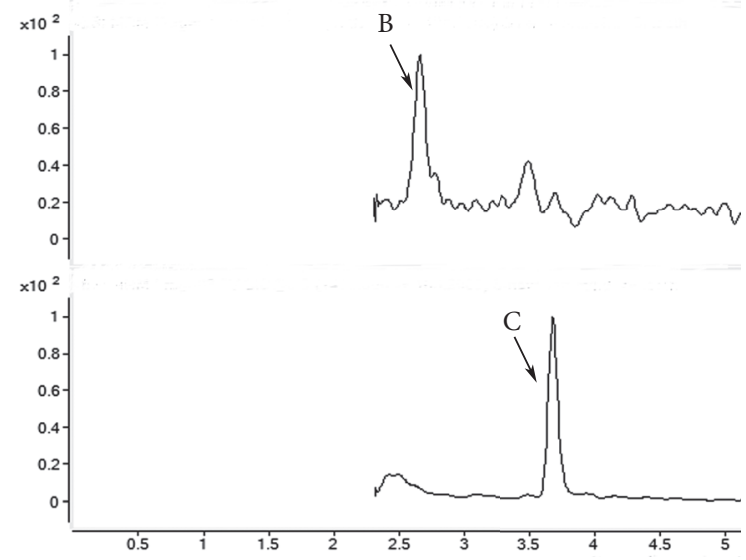

(c)

FIGURE 1: Mass spectrometer HPLC analysis of the reference standards (a), DSD (b), and DSD-Containing Serum (DSD-CS) (c). The peaks corresponding to puerarin (A), ferulic acid (B), and cinnamic acid (C) were identified.

treatment, MST1 cells $\left(5 \times 10^{4}\right.$ cells) were counted and seeded on 96-well culture plates precoated with $50 \mu \mathrm{l}$ of Matrigel Matrix at $37^{\circ} \mathrm{C}$ for 30 minutes in advance. Images were taken using the fluorescent microscope 1 hour after seeding.
2.9. Western Blot Analysis. The MST1 cells were collected and lysed for protein extraction. Protein $(25 \mu \mathrm{g})$ was separated by $10 \%$ polyacrylamide gels and transferred to the nitrocellulose membrane. The membranes were blocked by $5 \%$ BSA in PBST 

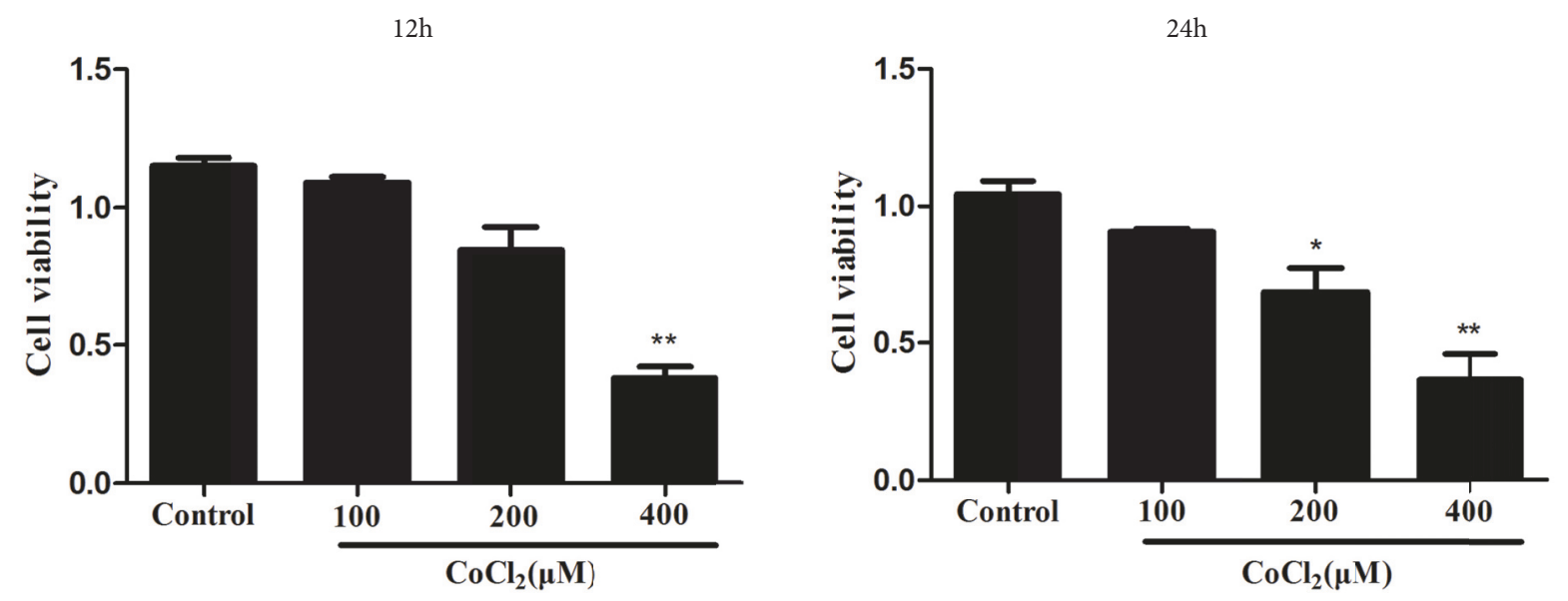

(a)
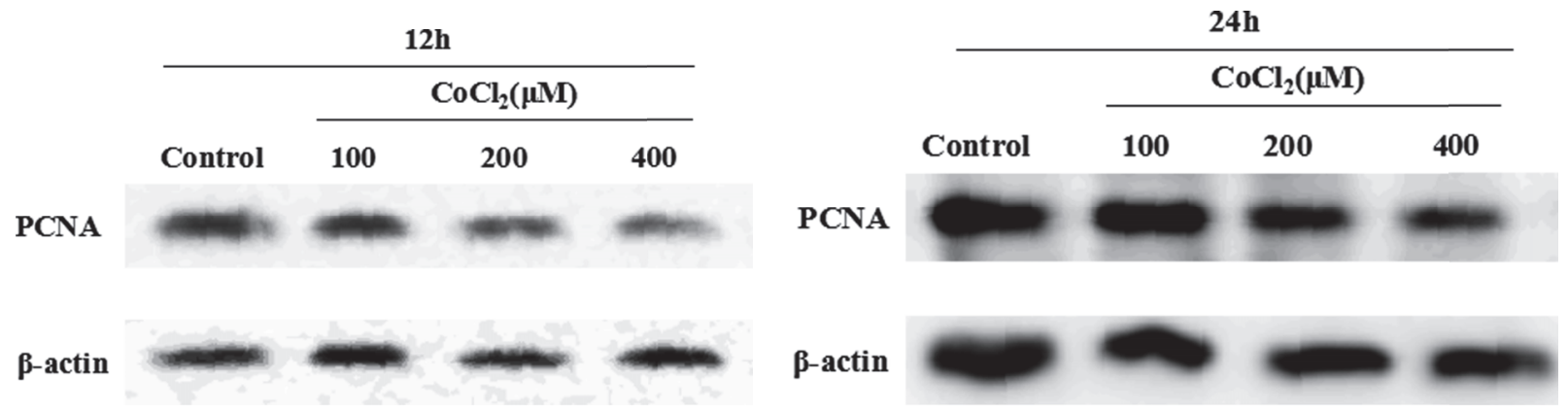

(b)
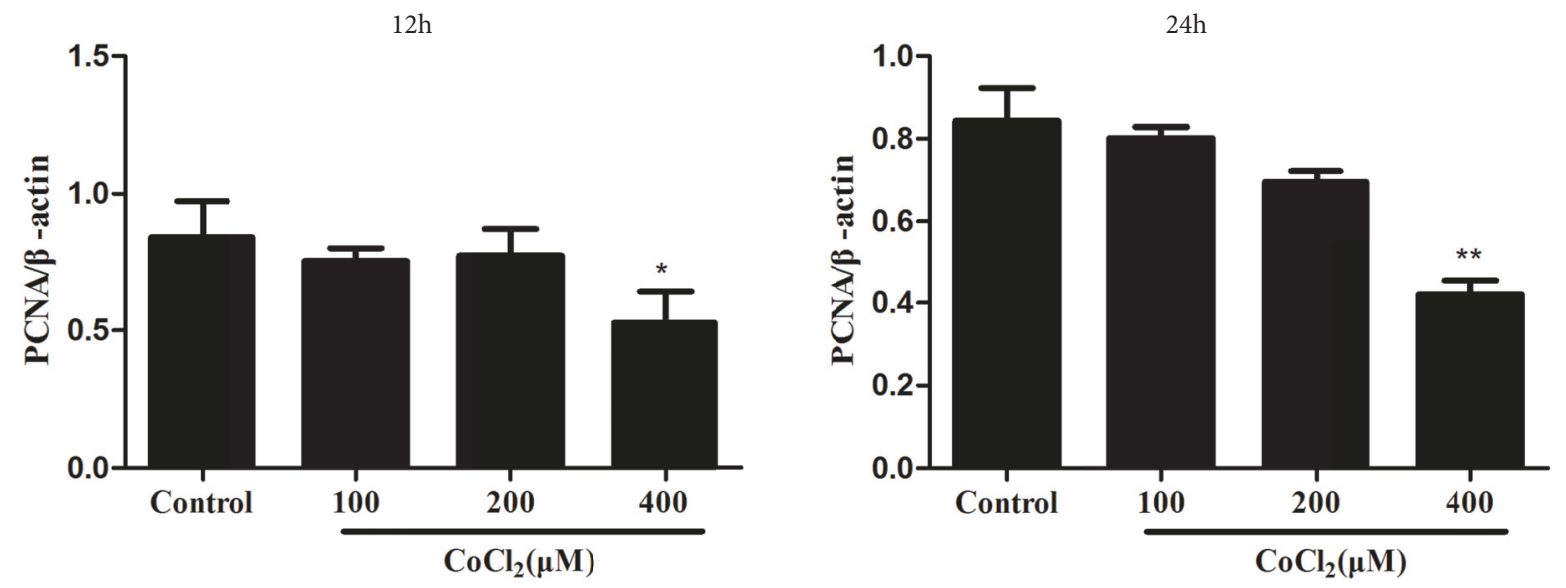

(c)

FIGURE 2: MST1 survival and proliferation were dose-dependently reduced by $\mathrm{CoCl}_{2}$ treatment. The cells were treated with CoCl $\mathrm{Canging}$ from 100 to $400 \mu \mathrm{M}$ for 12 hours or 24 hours and measured with CCK8 assay (a) and for PNCA protein level (b, c). ${ }^{\star} P<0.05$ and ${ }^{\star *} P<0.01$ versus Control group.

and incubated with primary antibodies overnight at $4^{\circ} \mathrm{C}$ followed by secondary antibody incubation at 1:5000 dilution for 1 hour at room temperature. The blots were developed in chemiluminescence (ECL) system and Kodak X-OMAT film.
2.10. Statistical Analysis. Results were presented as mean \pm SEM from three independent experiments at least. GraphPad Prism 5.0 software (GraphPad, CA, USA) was applied for data analysis. Statistical analysis among different groups was 


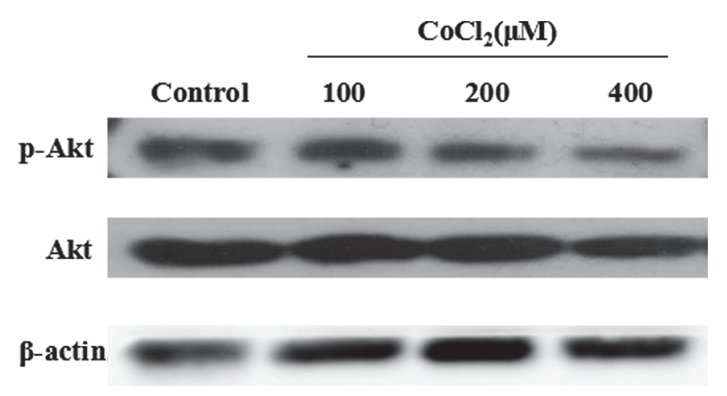

(a)

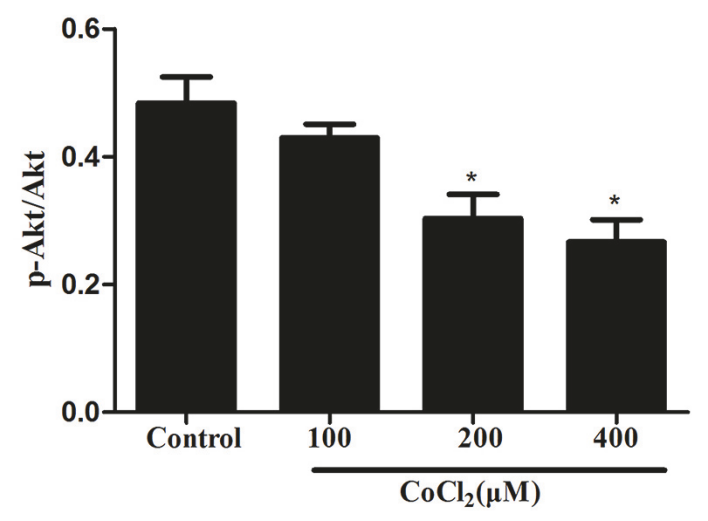

(b)

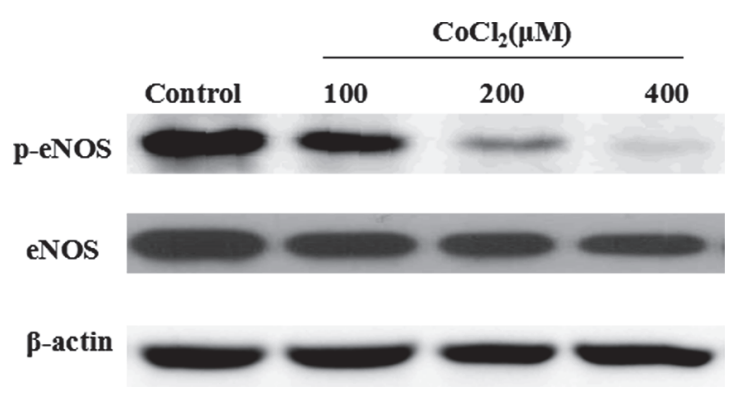

(c)

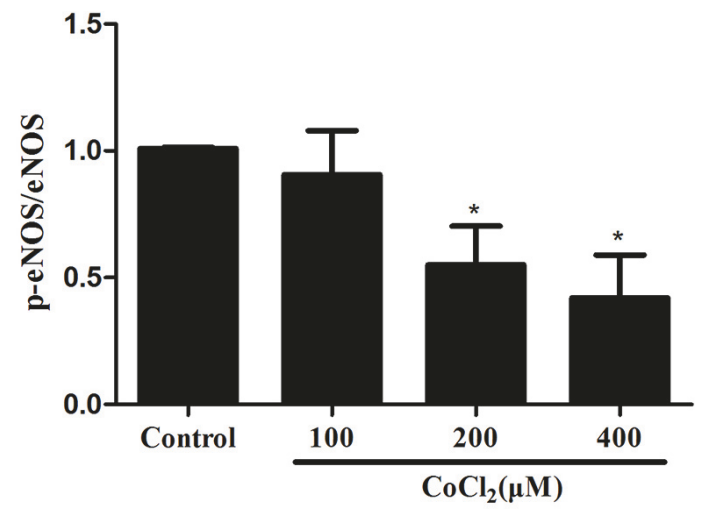

(d)

FIGURE 3: Phosphorylation of PI3K/Akt and eNOS was dose-dependently inhibited by $\mathrm{CoCl}_{2}$ treatment. The cells were treated with different concentrations of $\mathrm{CoCl}_{2}$ for 12 hours or 24 hours. Protein level of p-Akt was reduced in MST1 cells stimulated with CoCl $2(\mathrm{a}, \mathrm{b})$. Protein level of $\mathrm{p}$-eNOS was reduced in MST1 cells stimulated with $\mathrm{CoCl}_{2}(\mathrm{c}, \mathrm{d}) .{ }^{\star} \mathrm{P}<0.05$ versus control group.

carried out with the paired $t$-test. $P<0.05$ was considered statistically significant.

\section{Results}

3.1. Cobalt Chloride $\left(\mathrm{CoCl}_{2}\right)$ Dose-Dependently Reduced MST1 Survival and Proliferation. To investigate how MST1 respond to hypoxia stimuli, $\mathrm{CoCl}_{2}$ was applied in this study to mimic hypoxia status. As was shown in Figure 2, $\mathrm{CoCl}_{2}$ could damage cell viability dose-dependently as measured in Cell Counting Kit-8 (CCK8) assay. PCNA (proliferating cell nuclear antigen), identified as a marker of DNA synthesis during cell cycling, also decreased upon hypoxia stimulation indicating that MST1 proliferation was inhibited. These results suggested that $\mathrm{CoCl}_{2}$ could inhibit MST1 cell survival and proliferation capacity. $\mathrm{CoCl}_{2}$ stimulation at $200 \mu \mathrm{M}$ for 24 hours was determined for the rest of this study.

3.2. Cobalt Chloride $\left(\mathrm{CoCl}_{2}\right)$ Dose-Dependently Inhibited Phosphorylation of PI3K/Akt and eNOS. PI3K/Akt/eNOS signaling plays a pivotal role in endothelial cell biological functions [27-30]. Here we measured p-Akt, Akt, p-eNOS, and eNOS expression by Western blot. As is shown in Figure 3, $\mathrm{CoCl}_{2}$ could reduce p-Akt and p-eNOS. The result suggested that PI3K/Akt/eNOS signaling pathway was correlated to $\mathrm{CoCl}_{2}$ induced MST1 dysfunction.
3.3. DSD-CS Protected Cell Survival and Proliferation from Hypoxia-Induced Damage is PI3K/Akt Dependent. To determine whether DSD-CS could protect cell survival from hypoxia-induced damage, CCK8 and TUNEL assay were applied in this study. As presented in Figure 4, exposure to $200 \mu \mathrm{M} \mathrm{CoCl}_{2}$ for 24 hours significantly reduced cell survival compared with the vehicle group. The decrease of cell survival was abrogated by DSD-CS treatment, which was attenuated by PI3K/Akt inhibitor LY294002. Furthermore, we measured cell proliferation with Cell-Light ${ }^{\mathrm{TM}}$ EdU assay and PCNA expression. As shown in Figure 4, compared with the vehicle group, $\mathrm{CoCl}_{2}$ treatment significantly reduced cell proliferation, which was partially restored by DSD-CS. The reversed cell proliferation by DSD-CS was attenuated by LY294002. These results suggested that DSD-CS protected cell survival and proliferation from hypoxia-induced damage, in which PI3K/Akt signaling was involved.

\subsection{DSD-CS Protected MST1 Tube Formation Capacity} from Hypoxia-Induced Damage Was PI3K/Akt Dependent. A Matrigel tube formation assay was performed to measure the morphology and tube formation capacity of MST1. Compared to the vehicle group, $\mathrm{CoCl}_{2}$ treatment significantly reduced the amount of tubes formation, which was antagonized by DSD-CS. The reversed tube formation by DSDCS was blocked by PI3K/Akt inhibitor LY294002, suggesting 


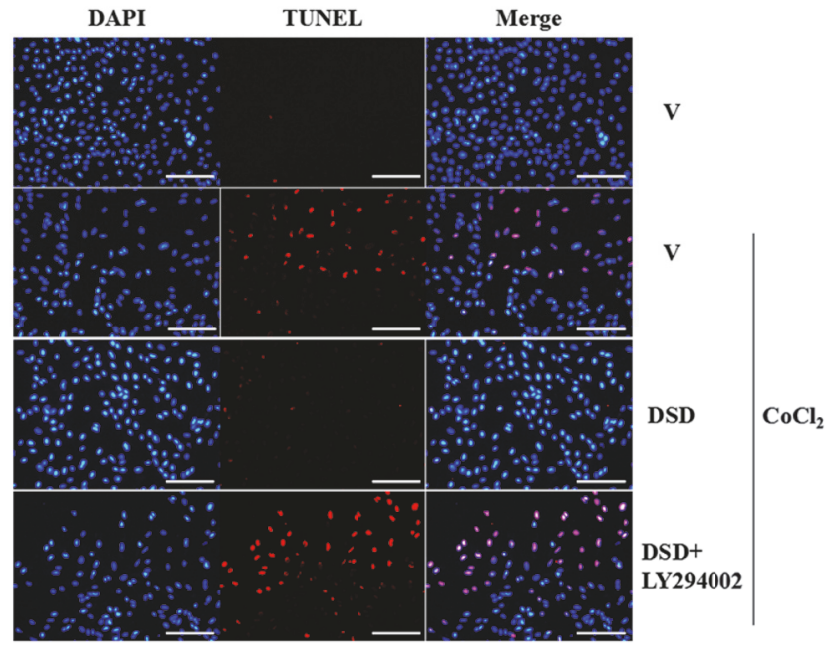

(a)

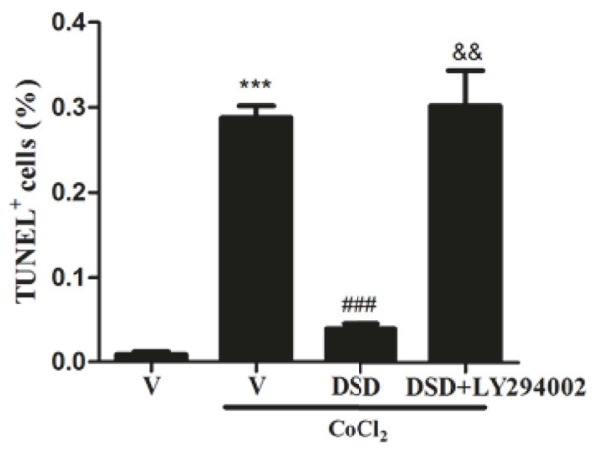

(b)

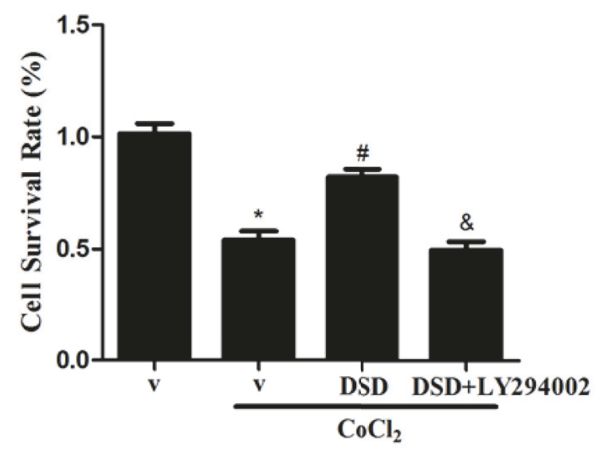

(c)

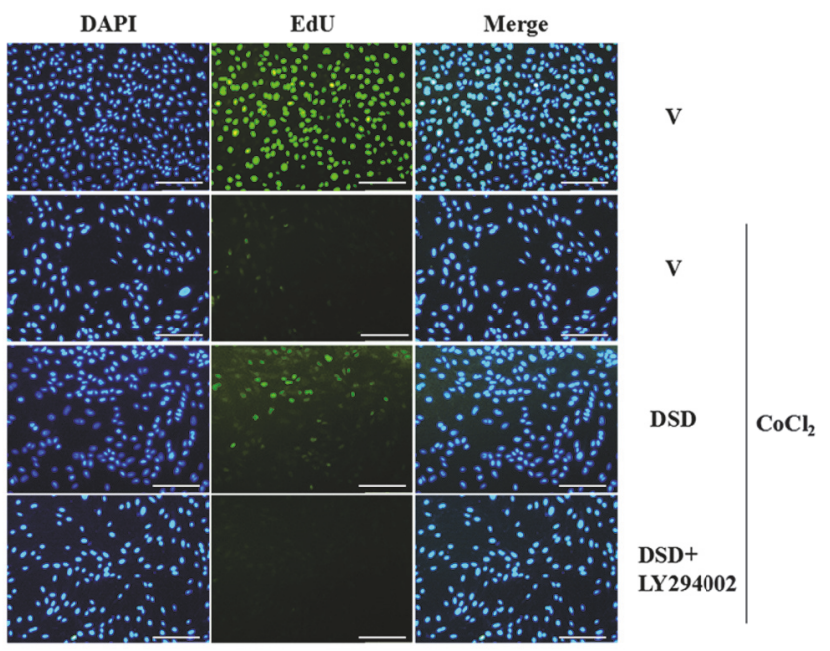

(d)

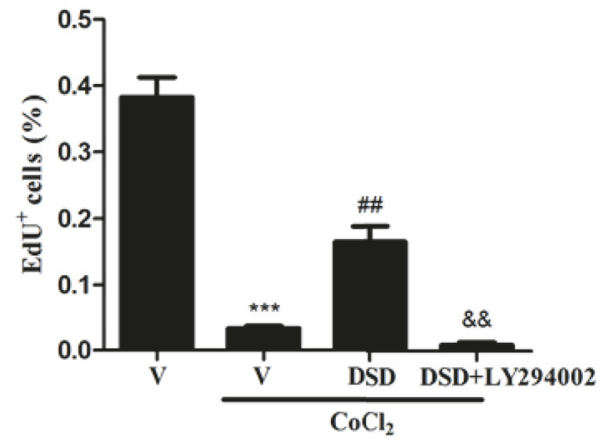

(e)

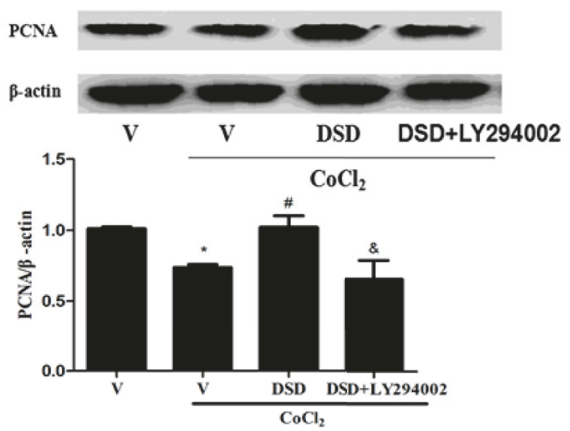

(f)

FIgure 4: Cell survival and proliferation were protected by DSD-CS from hypoxia-induced damage. (a-c) The cell survival capacity was measured by TUNEL assay and CCK8 assay. ${ }^{\star} P<0.05$ and ${ }^{* *} P<0.001$ versus vehicle group $(\mathrm{V}) ;{ }^{\#} P<0.05$ and ${ }^{\# \# \#} P<0.001$ versus $\mathrm{CoCl} \mathrm{C}_{2}+$ vehicle group $(\mathrm{V}) ;{ }^{8} P<0.05$ and ${ }^{\& \&} P<0.01$ versus $\mathrm{CoCl}_{2}+\mathrm{DSD}$ group. (d-f) The cell proliferation was measured by EdU assay and PCNA expression. ${ }^{\star} P<0.05$ and ${ }^{* \star *} P<0.001$ versus vehicle group $(\mathrm{V}) ;{ }^{\#} P<0.05$ and ${ }^{\# \#} P<0.01$ versus $\mathrm{CoCl}_{2}+$ vehicle group (V); ${ }^{8} P<0.05$ and ${ }^{\& \&} P<0.01$ versus $\mathrm{CoCl}_{2}+$ DSD group. Scale bars: $200 \mu \mathrm{m}$.

that PI3K/Akt signaling is involved in DSD-CS mediated improvement of cell tube formation capacity (Figure 5).

\subsection{DSD-CS Mediated Protective Action Activated PI3K/Akt/} eNOS Signaling. To investigate whether the effect of DSDCS was mediated by PI3K/Akt/eNOS signaling pathway, we measured p-Akt, Akt, p-eNOS, and eNOS expression by
Western blot. As shown in Figure 6, hypoxia stimulation reduced level of p-Akt compared to the control group. DSDCS treatment can partially antagonize the reduction. The effect was blocked by LY294002. Meanwhile, we found that $\mathrm{CoCl}_{2}$ reduced p-eNOS protein level in MST1 after 24 hours of incubation, which was abrogated by DSD-CS. Similarly, LY294002 could also block the reverse effect of DSD-CS 


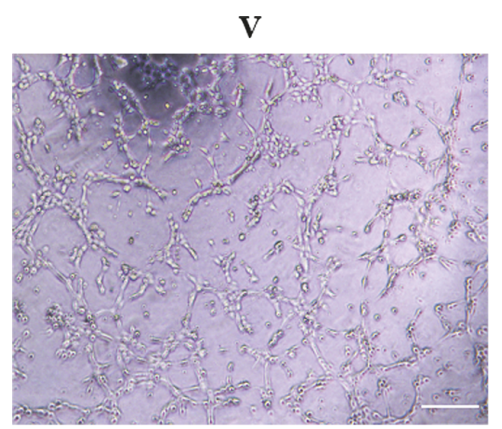

\section{$\mathrm{DSD}+\mathrm{CoCl}_{2}$}

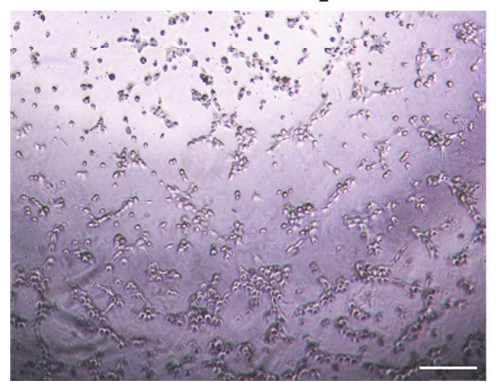

(a)

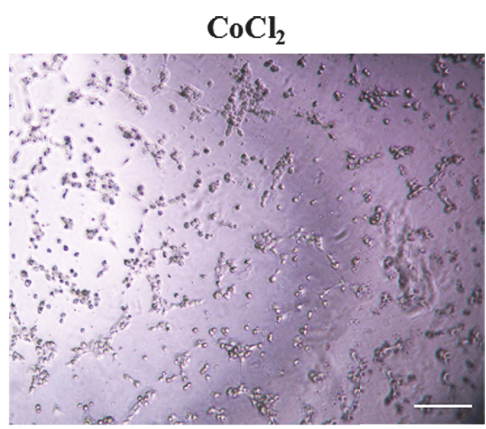

\section{$\mathrm{DSD}+\mathrm{CoCl}_{2}+\mathrm{LY} 294002$}

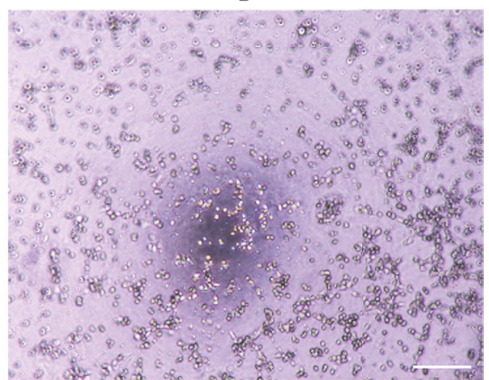

(a)

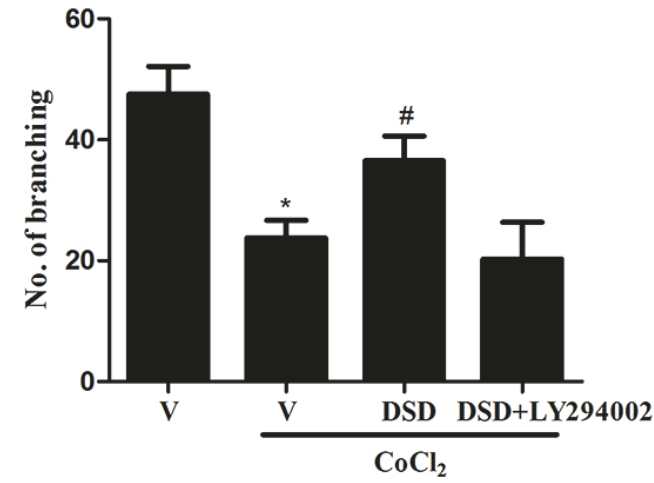

(b)

FIGURE 5: DSD-CS protected MST1 tube formation capacity from hypoxia-induced damage and was PI3K/Akt dependent. (a) The cells tube formation was measured by Matrigel tube formation assay. (b) Number of branching was quantified. ${ }^{\star} P<0.05$ versus vehicle group (V); ${ }^{\sharp} P<$ 0.05 versus $\mathrm{CoCl}_{2}+$ vehicle group (V). Scale bars: $200 \mu \mathrm{m}$.

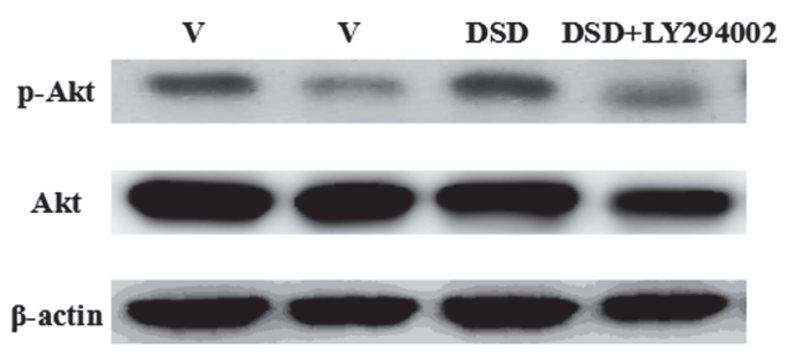

(a)

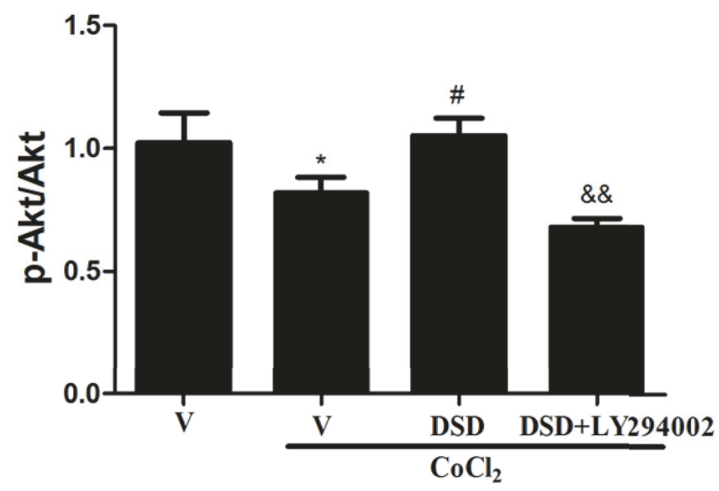

(b)

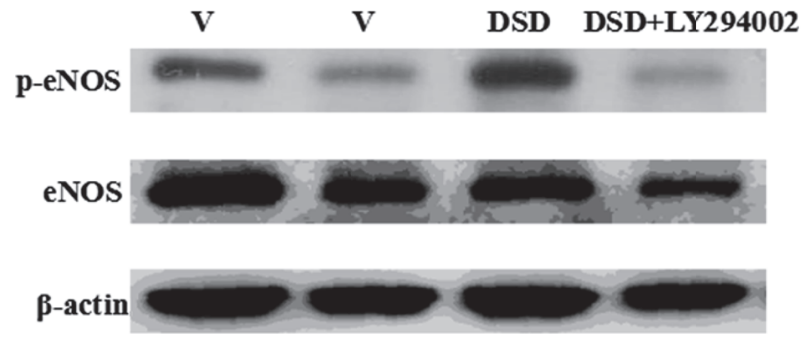

(c)

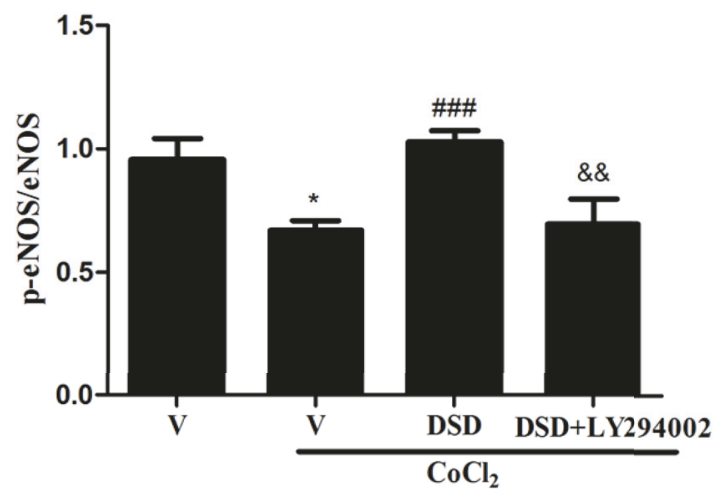

(d)

FIGURE 6: Effects of DSD on PI3K/Akt/eNOS signaling in $\mathrm{CoCl}_{2}$ treated MST1. (a-b) Representative Western blots of total and phosphorylated Akt protein expression. ${ }^{\star} P<0.05$ versus vehicle group $(\mathrm{V}) ;{ }^{\#} P<0.05$ and ${ }^{\# \# \#} P<0.001$ versus $\mathrm{CoCl}_{2}+$ vehicle group $(\mathrm{V})$; ${ }^{8} P<0.05$ and ${ }^{\& \&} P$ $<0.01$ versus $\mathrm{CoCl}_{2}+\mathrm{DSD}$ group; (c-d) Representative Western blots of total and phosphorylated eNOS protein expression. ${ }^{\star} P<0.05$ versus vehicle group $(\mathrm{V}) ;{ }^{\#} P<0.05$ and ${ }^{\# \# \#} P<0.001$ versus $\mathrm{CoCl}_{2}+$ vehicle group $(\mathrm{V}){ }^{*} P<0.05$ and ${ }^{\& \&} P<0.01$ versus $\mathrm{CoCl}_{2}+\mathrm{DSD}^{\mathrm{g}} \mathrm{group}$. 
indicating the involvement of Akt pathway in DSD-CS effect.

\section{Discussion}

Danggui Sini decoction (DSD) is a commonly used Chinese traditional medicine in increasing cardiovascular and peripheral circulation [1]. Previous researches had demonstrated that it is effective in treating vascular diseases, including Raynaud's phenomenon (RP), shock, heart failure, and severe poor extremity circulation [1-5]. Furthermore, its major components including Angelica sinensis, Ramulus Cinnamomi, and Radix Puerariae have been shown to possess great potential in promoting angiogenesis $[1,3,5,7]$ and activating antioxidant enzymes and anti-inflammation [814]. Here we showed that DSD greatly protected endothelial cell survival, proliferation, and function under hypoxic conditions.

Mounting studies have revealed the underlying mechanisms of Danggui Sini decoction (DSD) in regulating microcirculation. It was demonstrated that DSD could regulate the lipid metabolism, energy, and amino acid to adjust the fiber protease, platelet aggregation, and the expression of tissue factor [31]. In addition, DSD could alleviate diabetes-induced neuropathic pain by suppressing inflammatory process and gliosis in spinal cord [6]. Pancreas islet has a plenty of microvasculature transporting oxygen and hormones, which plays pivotal roles in supporting and regulating the proliferation and survival of pancreas $\beta$-cells [20-22]. It may also influence pancreatic islet transplantation, the responsive capacity to insulin resistance of $\beta$-cells, and the overall islet microenvironmental homeostasis $[32,33]$. Our present study demonstrated that DSD could efficiently protect islet derived endothelial cell-line-MST1 against Cobalt chloride $\left(\mathrm{CoCl}_{2}\right)$ induced lesions, including cell viability and proliferation and tube formation.

Endothelial cell viability, proliferation and tube formation capacity could be regulated by mitotic spindle dynamics [3436], the activation of proapoptotic factors, and the inhibition of antiapoptotic factors [37-39]. PI3K/Akt is reported to be a key signaling pathway involved in regulating endothelial cells viability, proliferation, and angiogenesis [40, 41]. One of its downstream targets is eNOS, which is also a critical regulator in endothelial cell survival and proliferation. By using MST1 cells, the phosphorylation of eNOS was completely blocked in the presence of LY294002 (a specific PI3K/Akt inhibitor). Besides, our study discovered that DSD-CS could reverse the $\mathrm{CoCl}_{2}$-induced PI3K/Akt/eNOS inhibition, which was blocked by the specific PI3K/Akt inhibitor LY294002, suggesting that DSD-CS protection of MST1 cell survival from hypoxia was mediated by PI3K/Akt/eNOS pathway.

Lining in the inner surface of blood microvessels, the vascular endothelium acts as the first interface for circulating blood components and regulates the perfusion and blood delivery. Besides, it interacts with extravascular tissues as well as other cell types lining along vasculatures. As a semipermeable barrier, it controls blood-tissue exchange of oxygen, nutrients, and hormones. Upon barrier dysfunction, it will lead to multiple organ dysfunction including metabolic disorder, infection, trauma, and other kinds of disease [42]. Our study demonstrated that DSD-CS treatment could efficiently protect MST1 against hypoxic injuries by maintaining cell viability and proliferation and tube formation capacity indicating that DSD treatment had a direct protection effect on islet endothelial cell.

In summary, we revealed that DSD is effective in protecting the islet endothelial cells from hypoxia-induced damage. DSD protection of endothelial cell survival and proliferation and tube formation capacity involved PI3K/Akt/eNOS pathway. In addition to its multiple roles in treating ischemiarelated diseases [1-6], DSD was showed here that it had a direct and potent effect in protecting cultured islet endothelial cell under hypoxic conditions, implying a further application in ameliorating islet microvasculature and microcirculation dysfunction when the hypoxia is present.

\section{Data Availability}

The data used to support the findings of this study are available from the corresponding author upon request.

\section{Disclosure}

All coauthors have participated in and agreed with the content and conclusions.

\section{Conflicts of Interest}

There are no potential conflicts of interest.

\section{Acknowledgments}

This work was supported by Xiamen Academician Workstation and National Natural Science Foundation of China for S. Liu (81270901), S. Yang (81673661), X. Li (81570770), and Caoxin Huang (81400419).

\section{References}

[1] Y.-J. J. Wu, S.-F. Luo, S.-H. Yang, J.-Y. Chen, K.-H. Yu, and L.-C. See, "Vascular response of Raynaud's phenomenon to nifedipine or herbal medication (duhuo-tisheng tang with danggui-sini tang): a preliminary study," Chang Gung Medical Journal, vol. 31, no. 5, pp. 492-502, 2008.

[2] W.-H. You, P. Wang, M.-Q. Li, Y. Zhang, Y.-L. Peng, and F.-L. Zhang, "Therapeutic effects of modified Danggui Sini Decoction on plasma level of advanced glycation end products in patients with Wagner grade 0 diabetic foot: a randomized controlled trial," Zhong Xi Yi Jie He Xue Bao, vol. 7, no. 7, pp. 622-628, 2009.

[3] J.-P. Zheng, "Experience in clinical application of Danggui Sini Decoction," Zhong Xi Yi Jie He Xue Bao, vol. 3, no. 4, p. 289, 293, 2005.

[4] Q.-Q. Chen, X. Han, W.-M. Wang, L. Zhao, and A. Chen, "Danggui sini decoction ameliorates myelosuppression in animal model by upregulating Thrombopoietin expression," Cell Biochemistry and Biophysics, vol. 71, no. 2, pp. 945-950, 2014. 
[5] J.-M. Yao, L.-J. Yu, Q. Chen et al., "Comparison of ferulic acid content in Radix Angelicae Sinensis, Danggui-Buxue-Tang and Danggui-Sini-Tang," Experimental and Therapeutic Medicine, vol. 7, no. 5, pp. 1364-1368, 2014.

[6] M. Liu, Q. H. Qiang, Q. Ling et al., "Effects of Danggui Sini decoction on neuropathic pain: Experimental studies and clinical pharmacological significance of inhibiting glial activation and proinflammatory cytokines in the spinal cord," International Journal of Clinical Pharmacology and Therapeutics, vol. 55, no. 5, pp. 453-464, 2017.

[7] I. Majewska and E. Gendaszewska-Darmach, "Proangiogenic activity of plant extracts in accelerating wound healing-a new face of old phytomedicines," Acta Biochimica Polonica, vol. 58, no. 4, pp. 449-460, 2011.

[8] A. M. Reddy, J. H. Seo, S. Y. Ryu, Y. S. Kim, and K. R. Min, "Cinnamaldehyde and 2-methoxycinnamaldehyde as NF- $\kappa \mathrm{B}$ inhibitors from Cinnamomum cassia," Planta Medica, vol. 70, no. 9, pp. 823-827, 2004.

[9] L. K. Chao, K.-F. Hua, H.-Y. Hsu et al., "Cinnamaldehyde inhibits pro-inflammatory cytokines secretion from monocytes/macrophages through suppression of intracellular signaling," Food and Chemical Toxicology, vol. 46, no. 1, pp. 220-231, 2008.

[10] J. Y. Guo, H. R. Huo, B. S. Zhao et al., "Cinnamaldehyde reduces IL-1beta-induced cyclooxygenase-2 activity in rat cerebral microvascular endothelial cells," European Journal of Pharmacology, vol. 537, no. 1-3, pp. 174-180, 2006.

[11] H.-S. Lee, B.-S. Kim, and M.-K. Kim, "Suppression effect of Cinnamomum cassia bark-derived component on nitric oxide synthase," Journal of Agricultural and Food Chemistry, vol. 50, no. 26, pp. 7700-7703, 2002.

[12] F.-H. Zheng, P. Wei, H.-L. Huo et al., "Neuroprotective effect of gui zhi (ramulus cinnamomi) on ma huang- (herb ephedra) induced toxicity in rats treated with a ma huang-gui zhi herb pair," Evidence-Based Complementary and Alternative Medicine, vol. 2015, Article ID 913461, 9 pages, 2015.

[13] Y. Zhang, P. Wang, Y. Xu, X. Meng, and Y. Zhang, "Metabolomic analysis of biochemical changes in the plasma of high-fat diet and streptozotocin-induced diabetic rats after treatment with isoflavones extract of radix puerariae," Evidence-Based Complementary and Alternative Medicine, vol. 2016, Article ID 4701890, 12 pages, 2016.

[14] X. Fu-Liang, S. Xiao-Hui, G. Lu, Y. Xiang-Liang, and X. Hui$\mathrm{Bi}$, "Puerarin protects rat pancreatic islets from damage by hydrogen peroxide," European Journal of Pharmacology, vol. 529, no. 1-3, pp. 1-7, 2006.

[15] E. Durr, J. Yu, K. M. Krasinska et al., "Direct proteomic mapping of the lung microvascular endothelial cell surface in vivo and in cell culture," Nature Biotechnology, vol. 22, no. 8, pp. 985-992, 2004.

[16] J. E. Schnitzer, "Update on the cellular and molecular basis of capillary permeability," Trends in Cardiovascular Medicine, vol. 3, no. 4, pp. 124-130, 1993.

[17] C. K. Lam, T. Yoo, B. Hiner, Z. Liu, and J. Grutzendler, "Embolus extravasation is an alternative mechanism for cerebral microvascular recanalization," Nature, vol. 465, no. 7297, pp. 478-482, 2010.

[18] W. J. Powers, R. L. Grubb Jr., D. Darriet, and M. E. Raichle, "Cerebral blood flow and cerebral metabolic rate of oxygen requirements for cerebral function and viability in humans," Journal of Cerebral Blood Flow \& Metabolism, vol. 5, no. 4, pp. 600-608, 1985.
[19] E. L. Bearer and L. Orci, "Endothelial fenestral diaphragms: a quick-freeze, deep-etch study," The Journal of Cell Biology, vol. 100, no. 2, pp. 418-428, 1985.

[20] M. F. Hogan and R. L. Hull, "The islet endothelial cell: a novel contributor to beta cell secretory dysfunction in diabetes," Diabetologia, vol. 60, no. 6, pp. 952-959, 2017.

[21] X. Li, L. Yuan, G. Xu et al., "Effect of renin angiotensin system blockade on the islet microvessel density of diabetic rats and its relationship with islet function," Journal of Huazhong University of Science and Technology (Medical Sciences), vol. 29, no. 6, pp. 684-688, 2009.

[22] M. Johansson, G. Mattsson, A. Andersson, L. Jansson, and P.-O. Carlsson, "Islet endothelial cells and pancreatic $\beta$-cell proliferation: studies in vitro and during pregnancy in adult rats," Endocrinology, vol. 147, no. 5, pp. 2315-2324, 2006.

[23] V. Vaithilingam, J. Oberholzer, G. J. Guillemin, and B. E. Tuch, "Beneficial effects of desferrioxamine on encapsulated human islets-in vitro and in vivo study," American Journal of Transplantation, vol. 10, no. 9, pp. 1961-1969, 2010.

[24] T. Linn, D. Erb, D. Schneider et al., "Polymers for induction of revascularization in the rat fascial flap: application of vascular endothelial growth factor and pancreatic islet cells," Cell Transplantation, vol. 12, no. 7, pp. 769-778, 2003.

[25] T. Linn, J. Schmitz, I. Hauck-Schmalenberger et al., "Ischaemia is linked to inflammation and induction of angiogenesis in pancreatic islets," Clinical \& Experimental Immunology, vol. 144, no. 2, pp. 179-187, 2006.

[26] M. Enghofer, K. H. Usadel, O. Beck, and K. Kusterer, "Superoxide dismutase reduces islet microvascular injury induced by streptozotocin in the rat," American Journal of PhysiologyEndocrinology and Metabolism, vol. 273, no. 2, part 1, pp. E376E382, 1997.

[27] D. Fulton, J.-P. Gratton, T. J. McCabe et al., "Regulation of endothelium-derived nitric oxide production by the protein kinase Akt," Nature, vol. 399, no. 6736, pp. 597-601, 1999.

[28] A. Ahsan, G. Han, J. Pan et al., "Phosphocreatine protects endothelial cells from oxidized low-density lipoproteininduced apoptosis by modulating the PI3K/Akt/eNOS pathway," Apoptosis, vol. 20, no. 12, pp. 1563-1576, 2015.

[29] J. Guo, A. A. Chakraborty, P. Liu et al., "pVHL suppresses kinase activity of Akt in a proline-hydroxylation-dependent manner," Science, vol. 353, no. 6302, pp. 929-932, 2016.

[30] H.-H. Yang, Y. Chen, C.-Y. Gao, Z.-T. Cui, and J.-M. Yao, "Protective effects of MicroRNA-126 on human cardiac microvascular endothelial cells against hypoxia/reoxygenationinduced injury and inflammatory response by activating PI3K/Akt/eNOS signaling pathway," Cellular Physiology and Biochemistry, vol. 42, no. 2, pp. 506-518, 2017.

[31] H. Zheng, X. Qin, H. Song et al., “(1)H-NMR based metabonomic approach to evaluate anti-coagulant effect of Danggui Sini decoction," Zhongguo Zhongyao Zazhi, vol. 40, no. 20, pp. 4088-4093, 2015.

[32] C. Dai, M. Brissova, R. B. Reinert et al., "Pancreatic islet vasculature adapts to insulin resistance through dilation and not angiogenesis," Diabetes, vol. 62, no. 12, pp. 4144-4153, 2013.

[33] A. R. Pepper, R. Pawlick, A. Bruni et al., "Transplantation of human pancreatic endoderm cells reverses diabetes post transplantation in a prevascularized subcutaneous site," Stem Cell Reports, vol. 8, no. 6, pp. 1689-1700, 2017.

[34] E. A. Nigg, "Mitotic kinases as regulators of cell division and its checkpoints," Nature Reviews Molecular Cell Biology, vol. 2, no. 1, pp. 21-32, 2001. 
[35] P. Salaun, Y. Rannou, and P. Claude, "Cdk1, plks, auroras, and neks: The mitotic bodyguards," Advances in Experimental Medicine and Biology, vol. 617, pp. 41-56, 2008.

[36] A. Musacchio, "Spindle assembly checkpoint: The third decade," Philosophical Transactions of the Royal Society B: Biological Sciences, vol. 366, no. 1584, pp. 3595-3604, 2011.

[37] O. Kutuk and A. Letai, "Regulation of Bcl-2 family proteins by posttranslational modifications," Current Molecular Medicine, vol. 8, no. 2, pp. 102-118, 2008.

[38] K. Shimada, S. Matsuyoshi, M. Nakamura, E. Ishida, M. Kishi, and N. Konishi, "Phosphorylation of FADD is critical for sensitivity to anticancer drug-induced apoptosis," Carcinogenesis, vol. 25, no. 7, pp. 1089-1097, 2004.

[39] T.-H. Wang, H.-S. Wang, H. Ichijo et al., "Microtubuleinterfering agents activate c-Jun N-terminal kinase/stress- activated protein kinase through both Ras and apoptosis signalregulating kinase pathways," The Journal of Biological Chemistry, vol. 273, no. 9, pp. 4928-4936, 1998.

[40] Z.-J. Wang, F.-M. Zhang, L.-S. Wang, Y.-W. Yao, Q. Zhao, and X. Gao, "Lipopolysaccharides can protect mesenchymal stem cells (MSCs) from oxidative stress-induced apoptosis and enhance proliferation of MSCs via Toll-like receptor(TLR)-4 and PI3K/Akt," Cell Biology International, vol. 33, no. 6, pp. 665674, 2009.

[41] C. H. Ryu, S. A. Park, S. M. Kim et al., "Migration of human umbilical cord blood mesenchymal stem cells mediated by stromal cell-derived factor-1/CXCR4 axis via Akt, ERK, and p38 signal transduction pathways," Biochemical and Biophysical Research Communications, vol. 398, no. 1, pp. 105-110, 2010.

[42] S. Y. Yuan and R. R. Rigor, "Integrated systems physiology: from molecule to function to disease," in Regulation of Endothelial Barrier Function, Morgan \& Claypool Life Sciences, San Rafael, Calif, USA, 2010, Copyright (c) 2011 by Morgan \& Claypool Life Sciences. 


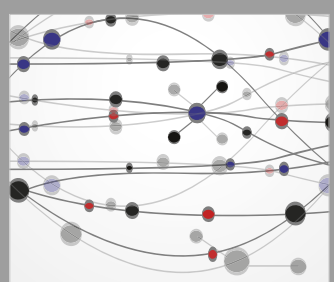

The Scientific World Journal
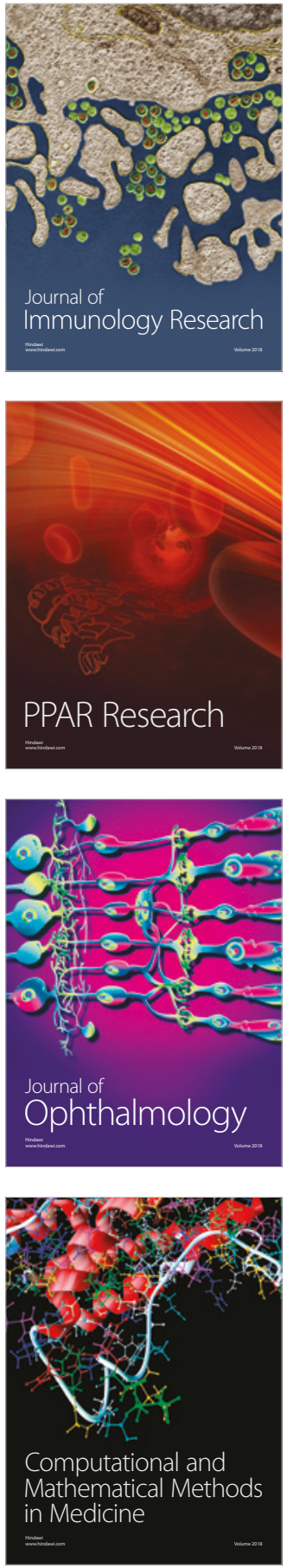

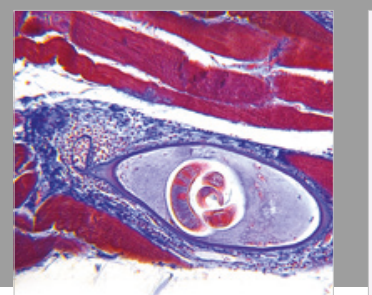

Gastroenterology Research and Practice

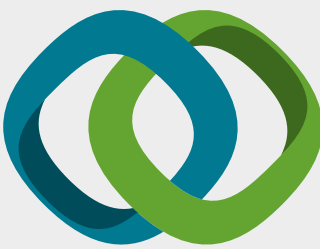

\section{Hindawi}

Submit your manuscripts at

www.hindawi.com
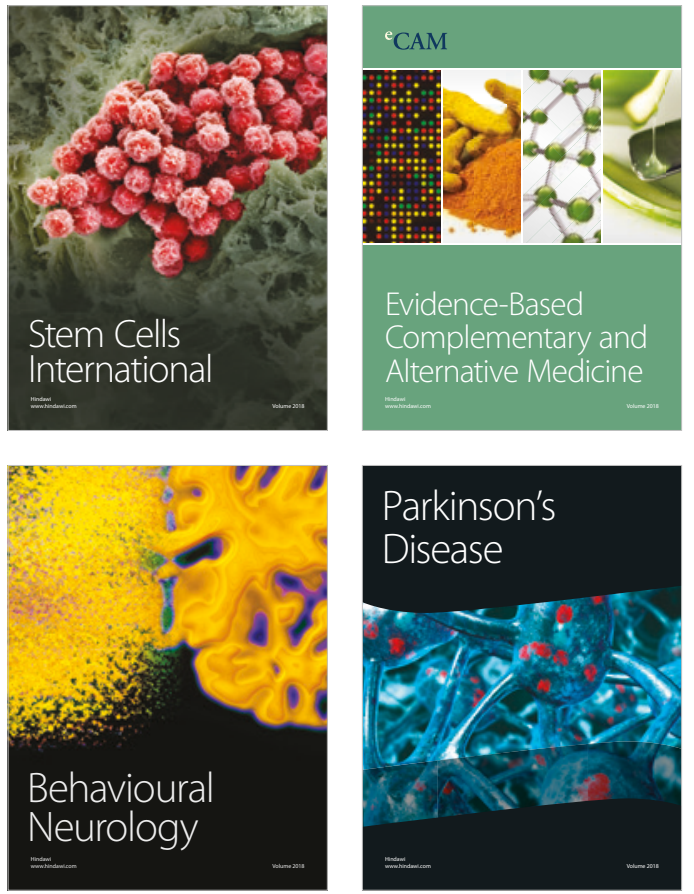

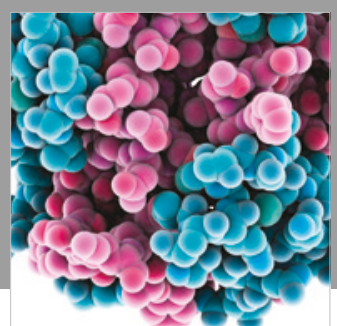

ournal of

Diabetes Research

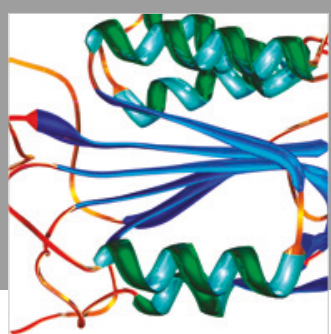

Disease Markers
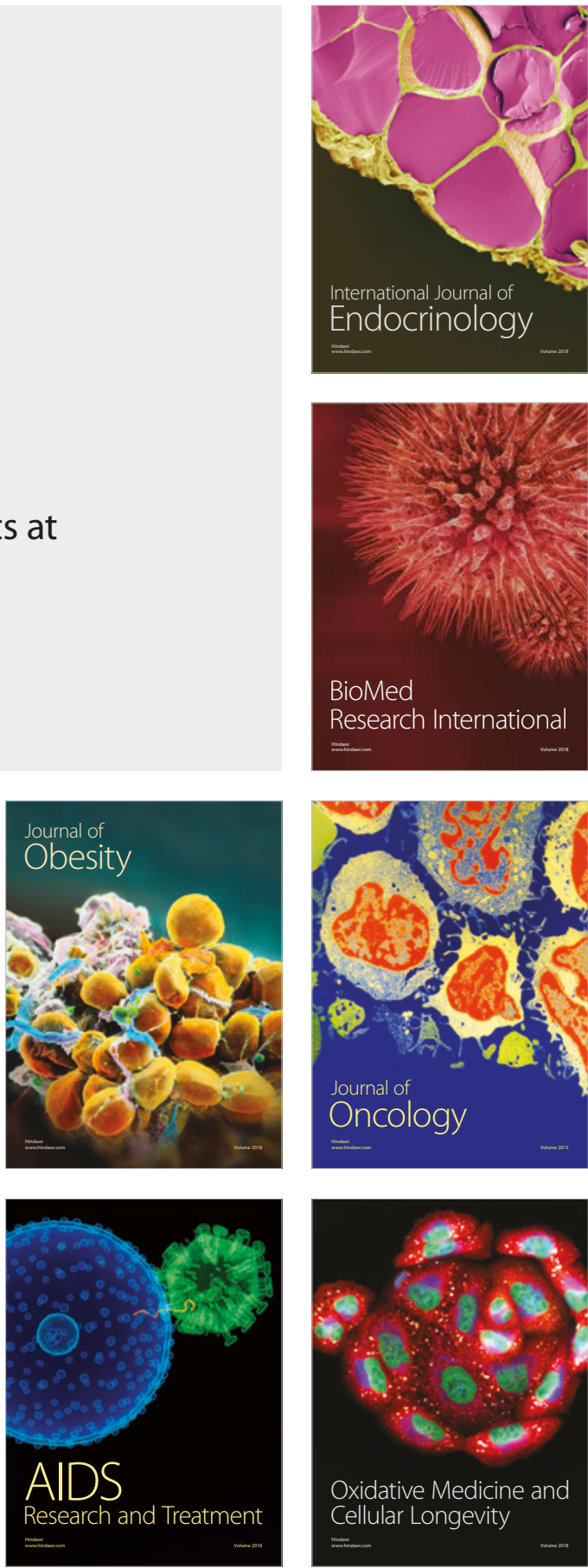